\title{
UTILIZAÇÃO DE LODO DE ESTAÇÃO DE TRATAMENTO DE ÁGUA RESIDUÁRIA DE CURTUME NOS PARÂMETROS MICROBIOLÓGICOS DO SOLO
}

\section{USE OF CURTUME RESIDUE WATER TREATMENT LODE IN THE SOIL'S MICROBIOLOGICAL PARAMETERS}

\author{
Isabella Santander de Souza ${ }^{1}$; Rita de Cássia Lima Mazzuchelli ${ }^{2}$; Fabio \\ Fernando de Araujo ${ }^{2}$. \\ ${ }^{1}$ Universidade do Oeste Paulista - UNOESTE - UNOESTE, Engenharia Ambiental e \\ Sanitária, Presidente Prudente, SP \\ E-mail: santander.souzaisa@gmail.com, rita@unoeste.br; fabioaraujo880@gmail.com
}

RESUMO - O presente trabalho tem o objetivo de avaliar as propriedades microbiológicas do solo que apresenta doses crescentes de lodo de água residuária de curtume. $\mathrm{O}$ experimento foi realizado em condições de casa de vegetação, localizada no Campus II - UNOESTE, na cidade de Presidente Prudente. Foi avaliado a aplicação de diferentes doses de lodo de água residuária de curtume nos parâmetros microbiológicos do solo. 0 estudo foi conduzido em 30 vasos de $10 \mathrm{Kg}$ durante o período de cem dias, após, foram coletadas amostras e encaminhadas ao laboratório para as avaliações biológicas no solo de carbono e nitrogênio da biomassa microbiana, e respiração basal do solo. Os dados foram submetidos à análise de variância, sendo realizado comparação por regressão. A aplicação da dosagem de $2 \mathrm{Mg}$ de lodo ha ${ }^{-1}$ sob a superfície do solo possibilitou um aumento no carbono e no nitrogênio da biomassa microbiana se mostrando eficiente. Dosagens superiores acabam reduzindo os elementos na biomassa.

Palavras-chave: biomassa microbiana; carbono; nitrogênio; respiração basal; ambiental.

ABSTRACT - The present work has the objective of evaluating the microbiological properties of the soil that presents increasing doses of tannery sludge. The experiment was carried out under greenhouse conditions, located at Campus II - UNOESTE, in the city of Presidente Prudente. The application of different doses of tannery sludge in microbiological soil parameters was evaluated. The study was conducted in 30 pots of $10 \mathrm{~kg}$ during the period of one hundred days, after which samples were collected and sent to the laboratory for the soil biological evaluations of carbon and nitrogen of the microbial biomass and basal respiration of the soil. The data were submitted to analysis of variance, and the comparison was made by regression. The application of the dosage of $2 \mathrm{Mg}$ of sludge ha-1 under the soil surface allowed an increase in the carbon and nitrogen of the microbial biomass being efficient. Higher dosages end up reducing the elements in the biomass.

Keywords: microbial biomass; carbon; nitrogen; basal respiration; environmental. 


\section{INTRODUÇÃO}

Um dos problemas ambientais relacionados a resíduos industriais está na reciclagem do couro bovino. Os resíduos deste ramo industrial apresentam elevadas cargas orgânicas e inorgânicas e de cromo, o qual é utilizado no processo de curtimento (SOUZA, 2009).

As características dos efluentes de curtumes variam de acordo com as matériasprimas e produtos químicos utilizados, bem como as etapas de processamento de couro (GUTTERRES et al., 2014). Segundo Konrad e Castilhos (2002), uma pele produz em média 15 kg de resíduos sólidos e, destes, 7,5 kg decantam no fundo da lagoa de tratamento primário na forma de lodo.

Visto como um dos principais problemas ambientais, são estimuladas alternativas adequadas de destinação desse resíduo sólido, buscando novas tecnologias. Uma das soluções para este passivo ambiental pode ser a aplicação desses resíduos no solo (FERREIRA et al., 2003; SILVA, 2009).

Para a utilização de um sub-produto, seus efeitos devem ser avaliados, a aplicação do lodo de curtume para a estrutura dos solos evidencia seu potencial como condicionador dos atributos físicos, com consequências importantes do ponto de vista ambiental relacionadas ao maior armazenamento de água e resistência à compactação e aos processos erosivos, que contribuem para a sustentabilidade das atividades agrícolas (ALVES et al., 2017), além de possuírem potenciais corretivos e de fertilizantes, propiciando a redução na aplicação de insumos minerais e melhoria da qualidade do solo (FERREIRA et al., 2003; SILVA, 2009).

A avaliação da atividade microbiana do solo, tem sido utilizada para assegurar a qualidade do insumo e a adequação do seu uso na agricultura (BOHM et al., 2014; ARMENTA et al., 2012; HERNÁNDEZ-ALLICA, 2006). A utilização de lodos provenientes das estações de tratamento de águas necessita de estudos avaliando os parâmetros microbiológicos como indicadores da poluição do solo, em virtude principalmente da maior sensibilidade dos microrganismos em detectar alterações de origens naturais e antropogênicas do solo. Consequentemente, o estudo da mineralização da carga orgânica de resíduos, estes estudos são de grande importância, para sua aplicação no solo, podendo nortear a definição da dose a ser aplicada a campo, que não prejudique a atividade microbiana no solo (MARTINES; ANDRADE; CARDOSO, 2006).

A biomassa microbiana do solo é responsável pela decomposição da matéria orgânica e ciclagem de nutrientes, funciona como um depósito de nutrientes para as plantas, e sua atividade reflete qualquer alteração feita no solo, seja ela benéfica, seja maléfica (GONÇALVES et al., 2009; BASTIDA et al., 2008).

As populações microbianas do solo sofrem acentuada influência do ambiente, podendo os microrganismos ou seus processos serem inibidos em até $100 \%$ por diversos fatores estressantes, como por exemplo a deposição de metais pesados (SIQUEIRA et al., 1994). Portanto, parâmetros microbiológicos são ferramentas bastante úteis no monitoramento da poluição do solo (JAHNEL et al., 1999), apontada como um sensível indicador de qualidade respondendo mais rapidamente do que parâmetros físicos e químicos (KASCHUK et al., 2010a; POWLSON et al., 1987).

Para uma maior sustentabilidade do resíduo a ser aplicada a campo, que não prejudique a processos produtivos, é importante o uso racional dos subprodutos das indústrias de beneficiamento de couro que representam para a agricultura um grande avanço nos processos de produção, ciclagem de nutrientes e consciência agroecológica, já que estes resíduos apresentam - aproveitamento no âmbito econômico, agronômico, industrial e social (BERILLI et al., 2015). Sendo assim, o presente trabalho tem o objetivo de avaliar as propriedades microbiológicas do solo que apresenta doses crescentes de lodo de águas residuais de curtume.

\section{METODOLOGIA}

O experimento foi realizado em condições de casa de vegetação, localizada no Campus II da Universidade do Oeste Paulista, UNOESTE, na cidade de Presidente Prudente, situada no estado de São Paulo, durante os meses de janeiro a junho de 2018. As coordenadas do local são latitude de $22^{\circ} 07^{\prime} 04^{\prime \prime}$ e longitude de $51^{\circ} 22^{\prime} 04^{\prime \prime}$ e altitude de aproximadamente 432 metros acima do nível do mar.

Foram utilizados vasos plásticos com capacidade de $12 \mathrm{~kg}$, nos quais foram 
adicionados o solo coletado na área experimental do campus II da Unoeste. Foram aplicadas as dosagens de lodo de curtume $0 ; 2 ; 4 ; 6$ e $8 \mathrm{Mg} \mathrm{ha}^{-}$ 1 , (calculadas através da área do vaso, correspondendo às aplicações de 0; 10,6; 21,2; 31,8 e 42,4 gramas por vaso, respectivamente). Constituindo um delineamento inteiramente casualizado com 6 repetições, totalizando 30 vasos. $\mathrm{O}$ lodo foi depositado na superfície do vaso e após um período de 30 dias, para a reação do mesmo ao solo, foram semeadas cinco sementes de milho, que após 15 dias foram desbastadas, deixando uma planta por vaso.

Ao final do experimento, aos 70 dias após a semeadura do milho, o solo foi coletado e encaminhado ao laboratório de biologia do solo da Unoeste, para as avaliações biológicas no solo de carbono e nitrogênio da biomassa microbiana (FERREIRA et al., 1999; TEDESCO et al., 1995) e respiração basal do solo (RODELLA; SABOYA, 1999).

Os dados foram submetidos à análise de variância (ANOVA) teste $F$, sendo realizado a comparação pela regressão, utilizando 0 programa estatístico Sisvar (FERREIRA, 2009).

\section{RESULTADO}

Podemos observar na figura 1 , que com a dosagem de $4 \mathrm{Mg}$ de lodo $\mathrm{ha}^{-1}$ sob a superfície do solo houve um crescimento do carbono da biomassa microbiana, que após esta dose o conteúdo de carbono da biomassa diminuiu se comparando ao tratamento sem a adição do resíduo (dose $0 \mathrm{Mg}$ ).

Figura 1. Carbono da Biomassa Microbiana do Solo - CBMS em mg.kg ${ }^{-1}$, com doses crescentes de lodo de águas residuais de curtume.

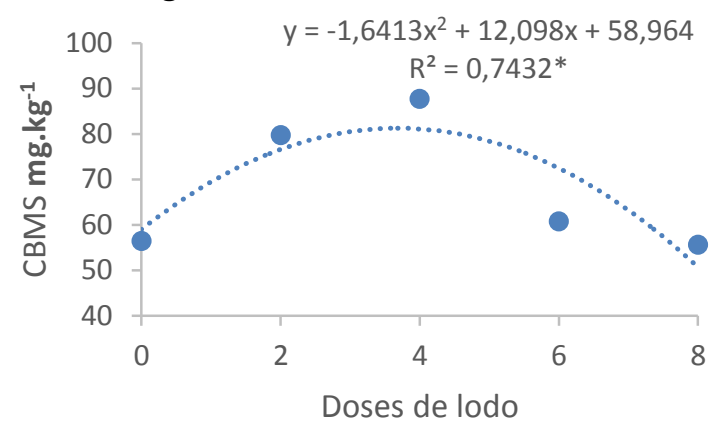

Em relação ao nitrogênio da biomassa microbiana do solo, podemos verificar (figura 2) que a dosagem de $2 \mathrm{Mg} \mathrm{ha}^{-1}$ possibilitou um aumento do elemento na biomassa, entretanto, dosagens superiores acabam reduzindo o elemento na biomassa microbiana.

Figura 2. Nitrogênio da Biomassa Microbiana do Solo - NBMS em $\mu^{-1}$ com doses crescentes de lodo de águas residuais de curtume.

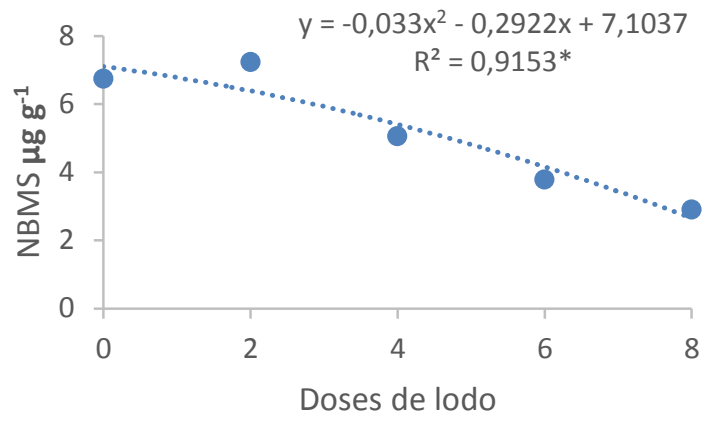

A respiração do solo não apresentou definição de comportamento em relação as doses de lodo aplicadas, a dose de 2 e $8 \mathrm{Mg} \mathrm{ha}^{-1}$, possibilitou uma menor liberação de $\mathrm{CO}_{2}$ para a atmosfera em relação as demais doses utilizadas, enquanto todas as doses utilizadas liberaram menores teores de $\mathrm{CO}_{2}$ quando comparadas a dose $0 \mathrm{Mg} \mathrm{ha}^{-1}$ (Figura 3).

Figura 3. Respiração basal do solo - RBS em $\mathrm{mg}$ de $\mathrm{CO}_{2} 48 \mathrm{~h}$, submetido a doses crescentes de lodo de águas residuais de curtume.

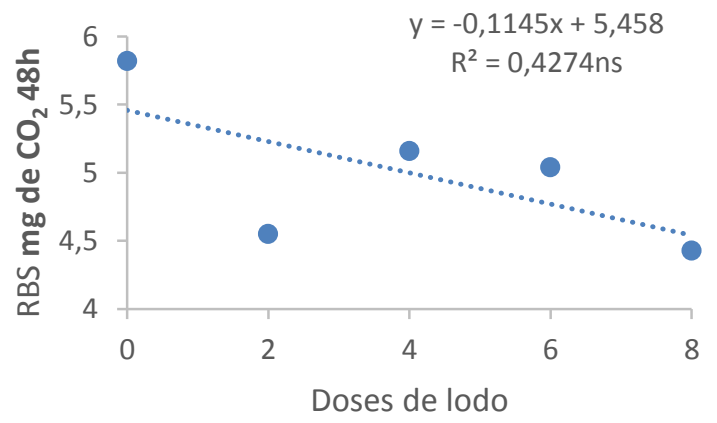

\section{DISCUSSÃO}

Pode-se observar no presente trabalho que a aplicação do resíduo lodo de estação de tratamento de água residuária de curtume no solo obteve variações nas taxas de CBMS, NBMS e não demonstraram influencia no comportamento da $\mathrm{RBS} \mathrm{CO}_{2}$.

Sendo de grande importância na reconstrução da fertilidade do solo, a microbiota fornece um indicativo precoce de mudanças no conteúdo de matéria orgânica (NETO; SOUZA, 2000). Assim, quando resíduos orgânicos são adicionados ao solo, os microrganismos utilizam o carbono (C) como fonte de energia e material orgânico microbiano, resultando num aumento 
da biomassa. Passianoto (2001), observou que o carbono e o nitrogênio estão entre os principais constituintes da matéria orgânica, e que a estimativa do carbono na biomassa microbiana indicou uma variação do mesmo em função dos tratamentos aplicados em sua pesquisa, onde o resíduo lodo do caleiro provou acréscimos no CBM em relação ao solo sem lodo, o que corroboram os resultados de PALÁCIO et al. (2000) que também constataram elevação no CBM em tratamentos que receberam o resíduo lodo do caleiro.

De acordo com Kaschuk et al. (2010) em sua pesquisa, o CBMS do tratamento com lodo solarizado foi bastante superior provavelmente em virtude do maior teor de nitrogênio $(N)$. $O$ que pode justificar o decréscimo do carbono da biomassa microbiano nas dosagens maiores que $4 \mathrm{Mg}$ de lodo $\mathrm{ha}^{-1}$ sob a superfície do solo, devido a redução significativa do nitrogênio da biomassa com dosagens superiores a $2 \mathrm{Mg} \mathrm{ha}^{-1}$.

Segundo Vieira e Cardoso (2003) em seus estudos, os dados relativos aos teores de $\mathrm{N}$ na biomassa microbiana do solo descartam a possibilidade de que grandes quantidades de $\mathrm{N}$ tenham sido imobilizadas pela microbiota do solo, à exceção da primeira coleta, em que os teores de $\mathrm{N}$ na biomassa microbiana foram maiores com a aplicação do lodo de esgoto. Esses resultados corroboram aos do presente trabalho onde apenas a dosagem de $2 \mathrm{Mg} \mathrm{ha}^{-1}$ possibilitou um aumento do elemento na biomassa.

Os resultados apresentados por Pereira (2017) mostram que, para os valores de CBMS, não houve diferença significativa entre os tratamentos na camada a $0-10 \mathrm{~cm}$ de profundidade, já na camada a $10-20 \mathrm{~cm}$, onde houve aplicação de lodo solarizado, os valores obtidos superaram aqueles encontrados no tratamento com adubação mineral. Ocorreu de forma similar aos resultados do presente trabalho, onde houve crescimento do carbono da biomassa microbiana nas dosagens de 2 e $4 \mathrm{Mg}$ de lodo ha ${ }^{-1}$ sob a superfície do solo.

De acordo com Pereira et al. (2017), a avaliação da RBS não resultou em diferença significativa entre tratamentos em seus resultados, provavelmente porque o lodo de esgoto é um substrato de rápida decomposição e, portanto, de maior liberação de nutrientes nos primeiros dias após sua aplicação no solo e posterior estabilização, como observado também por Armenta et al. (2012) em seu trabalho com lodo de esgoto aplicado em solo agrícola. $\mathrm{O}$ que pode fundamentar o não comportamento significativo da respiração do solo em relação as doses de lodos aplicadas no presente trabalho.

\section{CONSIDERAÇÕES FINAIS}

A aplicação da dosagem de $2 \mathrm{Mg}$ de lodo $h^{-1}$ sob a superfície do solo possibilitou um aumento no carbono e no nitrogênio da biomassa microbiana se mostrando eficiente. Dosagens superiores acabam reduzindo os elementos na biomassa.

\section{REFERÊNCIAS}

ALVES, André Divino et al. EFEITO DA ADIÇÃO DE LODO DE CURTUME NA AGREGAÇÃO DE UM LATOSSOLO CULTIVADO COM CANA DE AÇÚCAR. In: Anais do Congresso de Ensino, Pesquisa e Extensão da UEG (CEPE) (ISSN 2447-8687). 2017.

ARMENTA, R.; VACA, R.; LUGO, J.; AGUILA, P. del. Microbiological and biochemical properties of an agricultural Mexican soil amended with sewage sludge. Revista Brasileira de Ciência do Solo, v. 36, p. 1646-1655, 2012. https://doi.org/10.1590/S0100$\underline{06832012000500029}$

BALOTA, E.L.; COLOZZI Filho, A.; ANDRADE, D.S.; HUNGRIA, M. Biomassa microbiana e sua atividade em solos sob diferentes sistemas de preparo e sucessão de culturas. Revista Brasileira de Ciência do Solo, Viçosa, v.22, p.641-649, 1998.

BERILLI, S. S. et al. Utilização de lodo de curtume como substrato alternativo para produção de mudas de café conilon. Coffee Science, Lavras, v. 9, n. 4, p. 472- 479, 2014.

BOHM, G. B.; BETEMPS, G. R.; BIERHALS, L. N.; SANCHES FILHO, P. J.; SCHWANZ, S. M. Uso de lodo de estação de tratamento de esgoto em Argissolo cultivado com soja. Revista Thema, v. 11 p. 12-23, 2014. https://doi.org/10.15536/thema.11.2014.12$\underline{23.202}$

CASTILHOS, D.D.; TEDESCO, M.J.; VIDOR, C. Transformações biológicas do solo decorrentes da adição de resíduos de curtume e de cromo hexavalente. In: CONGRESSO BRASILEIRO DE CIÊNCIA DO SOLO, 27, 1999, Brasília. Resumo Expandido... Brasília, DF: SBCS, 1999, CD ROM. 
FERREIRA, Adao de Siqueira; CAMARGO, Flavio Anastacio de Oliveira; VIDOR, Caio. Utilização de microondas na avaliação da biomassa microbiana do solo. Revista Brasileira de Ciência do Solo, v. 23, n. 4, 1999.

FERREIRA FILHO, Sidney Seckler et al. Minimização da produção de lodo no tratamento de águas de abastecimento mediante uso do cloreto de polialumínio e sua disposição em estações de tratamento de esgotos. Engenharia Sanitaria e Ambiental, v. 14, n. 3, p. 317-326, 2009.

GONÇALVES, I. C. R.; ARAUJO, A. S. F.; CARVALHO, E. M. S.; CARNEIRO, R. F. V. Effect of paclobutrazol on microbial biomass, respiration and cellulose decomposition in soil. European Journal of Soil Biology, v. 45, p. 235-238, 2009. https://doi.org/10.1016/i.ejsobi.2009.01.002

GUTTERRES, M.; MELLA B. Chapter 16: Chromium in Tannery Wastewater, Book Heavy Metals in Water: Presence, Removal and Safety, Springer Publisher, Londres, 2014.

JAHNEL, M.C. Método de plaqueamento por gotas e outros parâmetros microbiológicos na avaliação da degradação de lodo ativado de curtume em solos. Piracicaba, 1997. 79p. Tese (Doutorado em AgronomiaSolos e Nutrição de Plantas) Escola Superior de Agricultura Luiz de Queiroz, USP, 1997.

MARTINES, Alexandre Martin; DE ANDRADE, Cristiano Alberto; CARDOSO, Elke Jurandy Bran Nogueira. Mineralização do carbono orgânico em solos tratados com lodo de curtume. Pesquisa Agropecuária Brasileira, v. 41, n. 7, p. 1149-1155, $2006 . \quad$ https://doi.org/10.1590/S0100204X2006000700011

NETO, F.C.M. da.; SOUZA, C.M. de. Avaliação de um método de recuperação de áres degradadas através da estimação da respiração da microbiota. In: REUNIÃO BRASILEIRA DE MANEJO E CONSERVAÇÃO DO SOLO E DA ÁGUA, 13, 2000, Ilhéus. Resumo Expandido... Ilhéus, Ba : SBCS, 2000, CD ROM.

PALÁCIO, S.M.; STANGARLIN, J.R.; SCHWANESTRADA, K.R.F.; FAGUNDES, M.R.S. da.; GALDINO, A.P.M.; SPESSATO, S. Avaliação da biodegradação de resíduos industriais através da taxa da respiração e biomassa microbiana. In: FERTBIO 2000 BIODINÂMICA DO SOLO, 2000, Santa Maria. Resumo Expandido... Santa Maria, RS: SBCS, 2000, CD ROM.

PASSIANOTO, Caio C. et al. Atividade e biomassa microbiana no solo com a aplicação de dois diferentes lodos de curtume. Current Agricultural Science and Technology, v. 7, n. 2, 2001.

PEREIRA, Najara Glenda Frota et al. EFEITOS DA APLICAÇÃO DE LODO DE ESGOTO ESTABILIZADO POR DIFERENTES PROCESSOS NOS ATRIBUTOS QUÍMICOS E MICROBIOLÓGICOS DE UM CAMBISSOLO HÁPLICO. Cadernos de Ciência \& Tecnologia, v. 32, n. 1/2, p. 115-127, 2017.

RODELLA, A. A.; SABOYA, L. V. Calibration for conductimetric determination of carbon dioxide. Soil Biology and Biochemistry, v. 31, n. 14, p. 2059- 2060, 1999. https://doi.org/10.1016/S0038- 0717(99)000462.

SIQUEIRA, J.O.; MOREIRA, F.M.S.; GRISI, B.M. et al. Microrganismos e processos biológicos dos solo: perspectiva ambiental. EMBRAPA-CNPAF, 1994. 142p. (Documentos,45).

SOUZA, M.R.F. Uso do lodo de curtume como fonte de nutrientes em plantas forrageiras. Programa de Pós-Graduação em Ciência Animal da Escola de Veterinária da Universidade Federal de Goiás, Goiânia, 2009.

VIEIRA, Rosana Faria; CARDOSO, Antonio Américo. Variações nos teores de nitrogênio mineral em solo suplementado com lodo de esgoto. Pesquisa Agropecuária Brasileira, v. 38, n. $7, \quad$ p. $867-874,2003$. https://doi.org/10.1590/s0100$\underline{204 \times 2003000700011}$

KASCHUK, G.; ALBERTON, O.; HUNGRIA, M. Three decades of soil microbial biomass studies in Brazilian ecosystems: Lessons learned about soil quality and indications for improving sustainability. Soil Biology and Biochemistry, v. 42 , n. 1, p. 1-13, 2010a. https://doi.org/10.1016/i.soilbio.2009.08.020

KASCHUK, G.; ALBERTON, O.; HUNGRIA, M. Quantifying effects of different agricultural land 
uses on soil microbial biomass and activity in Brazilian Biomes: Inferences to improve soil quality. Plant and Soil, v. 338, n. 1, p. 467-481, 2010b.

KONRAD, E.E.; CASTILHOS, D.D. Alterações químicas do solo e crescimento do milho decorrente da adição do lodo de curtume. Rev Bras Ciên Solo, v.26, p.257- 265, 2002. https://doi.org/10.1590/S0100-

$\underline{06832002000100027}$ 